\title{
Chronic Hypoxia Emerging as One of the Driving Forces behind Gene Expression and Prognosis of Hepatocellular Carcinoma
}

\author{
Jos F. van Pelt, Hannah van Malenstein, Frederik Nevens, and Chris Verslype \\ Liver Research Facility and Lab of Hepatology, University Hospital Gasthuisberg-Leuven, Herestraat 49, bus 703, 3000 Leuven, Belgium
}

Correspondence should be addressed to Jos F. van Pelt, jos.vanpelt@med.kuleuven.be

Received 24 March 2011; Accepted 12 April 2011

Academic Editors: A. B. Galosi, C. K. Panda, A. Wincewicz, and T. Yazawa

Copyright () 2011 Jos F. van Pelt et al. This is an open access article distributed under the Creative Commons Attribution License, which permits unrestricted use, distribution, and reproduction in any medium, provided the original work is properly cited.

Hepatocellular carcinoma (HCC) is one of the most frequent malignant tumors and an important cause of death. It has become evident that the tumor microenvironment, including hypoxia, plays a major role in the development of HCC. This paper focuses on the role of chronic hypoxia in HCC. Recently, we have shown the importance of chronic hypoxia on gene expression and behavior of liver cells. Using a cell culture model, we identified a distinct gene expression pattern and demonstrated clinical relevance for a 7-gene subset that can predict survival and early recurrence in patients. Recently, it was also shown that chronic hypoxia is associated with the upregulation of $\beta$-catenin and Hif $1 \alpha$ and that suppression of $\beta$-catenin reduced the metastatic potential of the tumor. Both studies demonstrate the importance of chronic hypoxia for the prognosis of HCC. Identifying the molecular pathways can help us to understand the mechanisms responsible for tumor aggressiveness.

\section{Introduction-Hepatocellular Carcinoma: The Clinical Problem}

Hepatocellular carcinoma (HCC) is one of the most frequent malignant tumors and an important cause of cancer death. Worldwide, it ranks fifth among the solid tumors and is the third cause of cancer-related mortality in males with $\sim 600.000$ deaths each year [1]. It is most prevalent in Asia (the annual incidence in China equals 100 per 100,000) and Africa. In Africa, HCC is mostly related to chronic hepatitis $B$ virus (HBV) infection in Asia to hepatitis B or C. The incidence in Europe and the USA is considerably lower with an annual incidence between 1 and 10 per 100.000 , but it is rising mainly due to hepatitis $\mathrm{C}(\mathrm{HCV})$ virus infection with an expected fivefold increase peaking around 2015 [2]. In more than $80 \%$ of cases, HCC is associated with cirrhosis or with advanced fibrosis [3,4] Cirrhosis is mostly due to chronic infection with the HBV or HCV, alcohol abuse, or metabolic disorders like hemochromatosis. More recently, nonalcoholic steatohepatitis (NASH) resulting from the insulin resistance syndrome is emerging as another important risk factor for cirrhosis and $\operatorname{HCC}[5,6]$.

Hepatocarcinogenesis is a multistep process in which a number of mutational genetic alterations accumulate in a cell. It involves the transition of a normal cell via the socalled initiated cell to a preneoplastic lesion that develops into malignant tumor and to clinical liver cancer [7].

Symptomatic HCC frequently runs a rapidly progressive clinical course and has a low rate of resectability and generally a poor response to nonoperative treatment and therefore a bad prognosis $[8,9]$. HCC in general develops over a long period (20-30 years) and is usually asymptomatic in the early stages. Therefore, a large group of patients have already an incurable disease at the time of diagnosis with the present available therapies. Identifying an HCC in a nodular (cirrhotic) liver represents a real challenge, especially if the lesion is smaller than two centimeters, which is an increasing problem due to the implementation of surveillance programs of patients at risk for HCC. HCC shows a variety of imaging features that reflect the variable pathological characteristics of this tumor. The cirrhotic liver harbors large regenerative nodules and dysplastic nodules (DNs), which may be very challenging to distinguish from small HCCs. The vascular supply to the lesion represents the key pathologic factor for differential diagnosis that is also reflected in imaging characteristics.

Through the progression from low-grade DN, to highgrade DN and finally early HCC, there is a development 
of arteries which become the dominant blood supply [7] This neoangiogenesis allows the imaging diagnosis of HCC. Imaging techniques may establish the diagnosis of HCC in nodules larger than two centimeters showing characteristic early arterial hypervascularisation on a dynamic imaging technique, even with a normal serum alpha-fetoprotein (AFP) value. In lesions ranging from one to two centimeters, two different dynamic imaging techniques should demonstrate the typical vascular features. If not, special workup for these lesions may consist of either followup or preferably obtaining a pathological proof using a safe approach. A lesion that is increasing in diameter, the presence of a tumor capsule, internal mosaic architecture, or invasion of portal vein branches are strongly suggestive of HCC [10].

Alpha-fetoprotein is the only serological marker commonly used in diagnosis and has a poor sensitivity ranging from $39 \%$ to $65 \%$ and a specificity ranging from $76 \%$ to $97 \%$. This high variability relates to the different cutoffs used in various (retrospective) studies. At values over $200 \mathrm{IU} / \mathrm{mL}$, AFP is reliable as tumor marker but the percentage of patients with such high levels is very small and false positives are quite frequently seen especially in viral cirrhosis. The availability of other suitable serological early diagnostic markers would be very useful. Some new tumor markers are emerging, such as human cervical cancer oncogene and human telomerase reverse transcriptase mRNA, and these have higher accuracies than AFP.

Furthermore, some other tumor markers, such as glypican-3 (GPC-3), Golgi Protein 73, vascular endothelial growth factor (VEGF), des-gamma-carboxyprothrombin (DCP), and transforming growth factor-betal, combined with AFP seem to have a greater sensitivity than AFP alone.

The treatment of patients with HCC is based on staging, which includes assessment of tumor extent, liver function, portal pressure, and clinical performance status. The widely used Barcelona-Clinic-Liver-Cancer (BCLC) staging system links staging of HCC in cirrhosis with treatment modalities $[8,9]$. The system identifies those patients with early HCC (stage 0 and A) who may benefit from curative therapies (resection, radiofrequency ablation, and liver transplantation), those at intermediate or advanced stage (stage B, C) who may benefit from palliative treatments (such as transarterial chemo- or radioembolisation), and the patients with a very poor life expectancy (stage D) where best supportive care is the only option $[9,11]$ (see Figure 1).

Until recently, patients with advanced hepatocellular carcinoma (BCLC-stage C) could only be offered best supportive care or systemic chemotherapy with an unfavorable risk-benefit ratio. The advent of targeted drugs has offered hope to our patients. Sorafenib is a multikinase inhibitor targeting the Raf serine/threonine kinases and the VEGFR13 , PDGFR- $\beta$, c-Kit, Flt3, and p38 tyrosine kinases, that block the VEGF and PDGF-dependent angiogenesis. The drug is now considered the standard of care in patients with advanced hepatocellular carcinoma and preserved liver function, based upon two phase III trials that showed an improved overall survival and progression-free survival compared to placebo. Median survival increased from 7.9 to 10.7 months (hazard ratio $0.69,95 \%$ CI $0.55-0.87$ ) $[12,13]$.
Most drug-related and manageable adverse events include fatigue, anorexia, diarrhea, and hand-foot skin reaction. Unfortunately, all patients with advanced HCC still die from the disease and there is an unmet need for other drugs, either as a single agent or in combination with sorafenib. A phase III clinical trial with sunitinib, a drug with a similar mode of action as sorafenib, has recently been stopped because of higher toxicity and no superior efficacy compared to sorafenib. Other clinical trials explore the safety and efficacy of everolimus that targets mTOR, which is a central regulator of cell growth and angiogenesis.

\section{Tumor Microenvironment}

Supported by a growing number of scientific reports, it has become clear over recent years that the tumor environment also plays an important role in tumorigenesis and tumor progression [14]. Cancer cells are not as autonomous as once thought; they depend on angiogenesis, inflammatory cells, and fibroblasts $[15,16]$. Obvious, in the cirrhotic liver there is an abundance of fibroblasts and there is emerging evidence that some are cancer-associated fibroblasts (CAFs) [17]. In HCC, the progression of malignant hepatocytes frequently depends on transforming growth factor (TGF)$\beta$ provided by the stromal cells (fibroblasts, macrophages, etc.). This TGF- $\beta$ is one of the factors that can induce an epithelial-to-mesenchymal transition (EMT) [18]. For different types of tumors, it has been demonstrated that an EMT switch is associated with a worse prognosis as seen in esophageal squamous cell carcinoma [19], gastric cancer cells [20], bladder cancer [21], nonsmall cell lung cancer [22], and pancreatic ductal adenocarcinoma [23]. For HCC, this correlation has also been found with an independent effect of Twist and Snail in promoting metastasis of hepatocellular carcinoma [24-27].

Characteristics for EMT are a shift in gene expression with an upregulation of Twist [23, 28], Snail [29], VEcadherin [30], Vimentin [31], downregulation of E-cadherin [32], hepatocyte transcriptional factor HNF4 $\alpha$, and changes in the cytoskeleton. Important molecular pathways involved in EMT are Wnt/ $\beta$-catenin signaling $[27,33]$ and through growth factor signaling like PDGF [34].

In EMT, there is a destabilization of adherent junctions [35] and the cells develop more invasive properties [31]. In mouse models, both in vitro and in vivo, epithelial-tomesenchymal transition has been shown to promote invasion and metastasis $[26,36]$.

Two major risk factors associated with HCC (hepatitis B virus and hepatitis $C$ virus) have been shown to contribute to EMT in HCC tumor progression $[37,38]$.

Kim et al. has shown that epithelial-to-mesenchymal transition gene signature can predict clinical outcome of hepatocellular carcinoma. They used RT-PCR to determine expression of EMT-related genes and could predict survival of patients using only four genes (E-cadherin (CDH1), inhibitor of DNA binding 2 (ID2), matrix metalloproteinase 9 (MMP9), and transcription factor 3 (TCF3) [39].

The role of hypoxia in initiation and progression of HCC is only partly understood. Hypoxia is involved in 


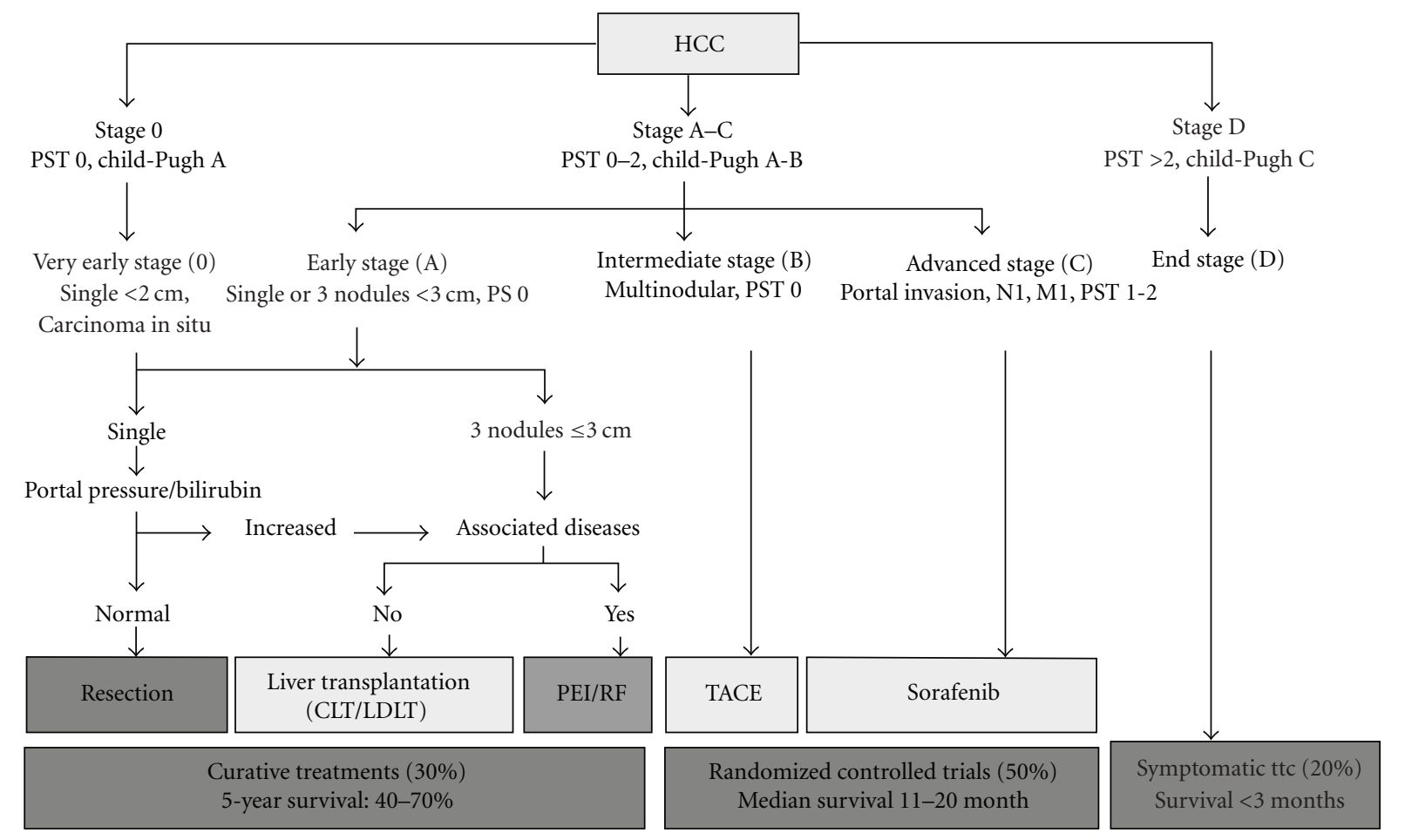

Figure 1: Barcelona Clinic Liver Cancer (BCLC) staging classification and treatment schedule (Adapted from [9]). PST: primary systemic therapy, CLT: cadaveric liver transplantation, LDLT: living donor liver transplantation, PEI: percutaneous ethanol injection, RF: radio frequency ablation, and TACE: transcatheter arterial chemoembolization.

neoangiogenesis and the formation of new blood vessels and it induces gene expression through the transcription factor Hif $1 \alpha$ that regulates more than 50 targets genes. Hypoxia through Hifl $\alpha$ [40] stimulates EMT [41, 42] involving PI3 kinase/AKT signaling [43-45] and redox mechanisms [46]. Hypoxia further influences the tumor microenvironment by stimulation of stellate cells, and it promotes fibrosis [42].

\section{Molecular Classification for HCC}

Several studies have tried to classify HCCs based on their gene expression or on their chromosomal alteration. Obtaining a molecular classification of hepatocellular carcinoma, however, remains a striking challenge because of the overwhelming genomic complexity of HCC [47]. Hitherto, there are several large studies published that classify HCC based on gene expression. Two of them could identify subgroups with common affected pathways such as $\beta$-catenin and proliferation signals $[48,49]$ and one group $[50,51]$ was able to correlate the subgroups with survival. Other studies tried to find common affected genes that are associated with prognostic factors such as vascular invasion and metastasis, but the overlap between these studies is very poor. Three groups studied the surrounding liver tissue, instead of the tumor tissue itself [52-54]. Based on their findings, late recurrence can be predicted in cirrhotic patients but these recurrences are probably "second primary tumors in an atrisk liver."
Combining all these studies led to the transcriptome classification as developed by Zucman-Rossi and Boyault $[48,55]$. A subgroup of patients have activation of pathways related to cell proliferation (IGF signaling, RAS/MAPK, and mTOR signaling) and differentiation (Wnt- $\beta$-catenin and Hedgehog). Overall, we can classify as follows: (1) a proliferation subgroup with Akt/mTOR activation, (2) the closely related DNA-repair/cell-cycle subgroup, (3) the $\beta$ catenin subgroup, (4) the immune-related subgroup, (5) the MAPK-/c-jun-/c-Myc-related genes and Jak/STAT-related genes, and (6) the metastasis-related genes group.

Molecular analysis also helped to identify important cellular pathways and with them possible therapeutic targets. The first molecule that in this way has shown clinical application for liver cancer is the multikinase inhibitor sorafenib, others are currently in different stages of clinical studies. The prognostic implication of this clustering based on pathways remains unclear.

\section{Hypoxia and HCC}

One of the factors that appears to affect cancer cell behavior and patient prognosis is hypoxia [56]. Hypoxia is associated with poor prognosis in several malignancies, such as cervix and breast carcinoma, and with the development of resistance to chemotherapeutic agents and radiation [57, 58]. And although HCC is a hypervascular malignancy, still there are regions with reduced oxygen supply [59]. Hypoxic regions are already present in the early stage 


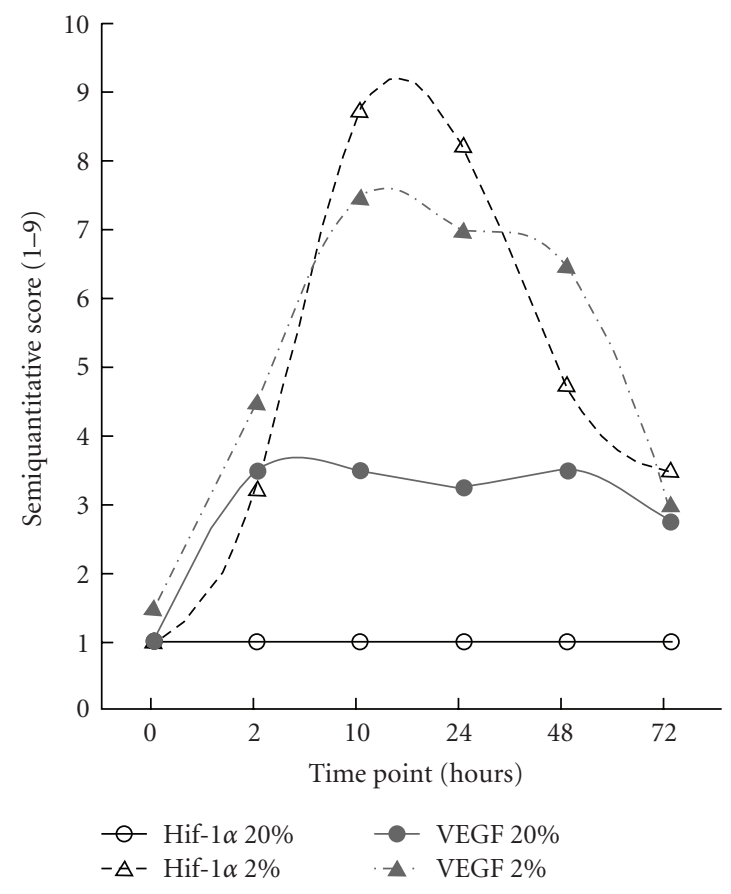

(a)

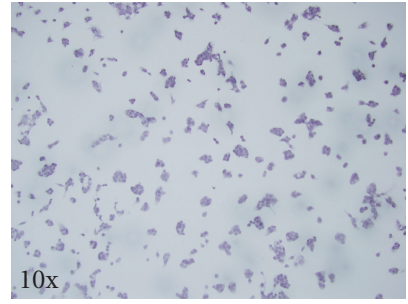

(b)

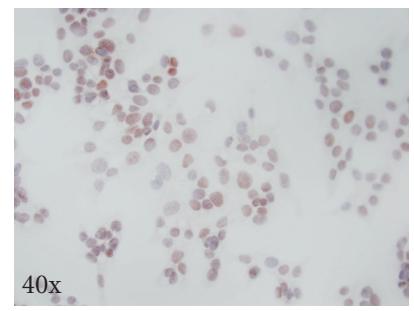

(c)

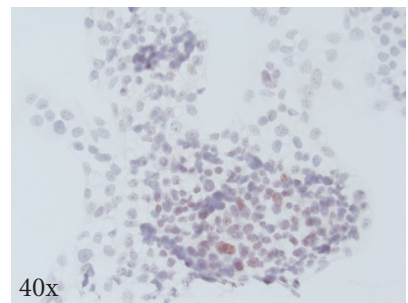

(d)

FIGURE 2: Immunohistochemical staining for Hif-1 $\alpha$ and VEGF after exposure to normal (20\%) or impaired (2\%) oxygen at several time points. (a) To evaluate the staining, a semiquantitative quickscore (1-9) was used which combines positivity (P) with a range from 1 to 6 and intensity (I) with a range from 0 to 3 . There is a strong induction of both proteins in the acute phase ( $0-24$ hours), but after prolonged hypoxia a new balance occurs. Hif- $1 \alpha$ is not expressed under normal oxygen (20\%) conditions, whereas VEGF has a low constitutional expression. (b-d) Immunohistochemical staining under hypoxic conditions: (b) Hif- $1 \alpha$ staining at 0 hrs-there is no Hif- $1 \alpha$ present; (c) Hif- $1 \alpha$ staining after $24 \mathrm{hrs}$ - almost all cells are positive; (d) Hif- $1 \alpha$ staining after $72 \mathrm{hrs}$ — some cells are positive.

when the vasculature is not sufficiently extended and in more advanced stages when rapid cell proliferation induces hypoxia [60]. Moreover, liver cancer usually develops in a cirrhotic environment where the blood flow is already impaired and, more importantly, during the expansion of the tumor, the neovascularization is unorganized with leaky blood vessels, arteriovenous shunting, large diffusion distances, and coiled vessels. These structural and functional defects lead to both acute hypoxia due to fluctuating flow and to chronic hypoxia due to diffusion distances of more than $150 \mu \mathrm{m}[61,62]$.

Hypoxia induces a transcription response that is mainly initiated by hypoxia inducible factor- 1 alpha $(\operatorname{Hif}-1 \alpha)$. In normoxic conditions, Hif- $1 \alpha$ is rapidly broken down in the cytoplasm through ubiquitination by the cooperation between Von Hippel Lindau protein and the oxygen sensors prolyl hydroxylase (PHD) and factor inhibiting Hif (FIH). When oxygen is lacking, Hif- $1 \alpha$ accumulates and can translocate to the nucleus and form the transcriptionally active complex Hif- 1 by coupling to Hif- $1 \beta$ (also ARNT). Hif- 1 is a master control gene with over fifty target genes and alters different pathways, such as angiogenesis (VEGF), glycolysis (GLUT1), apoptosis (BNIP), and cell proliferation (IGF2) among others [57]. Hitherto, studies evaluated only the early changes in gene expression of cells exposed to maximum 24 hours of hypoxia [63-65]. From in vitro studies, it has come forward that there is a relationship between mTOR inhibition and hypoxia [66]. Hypoxia can also elicit multidrug resistance to chemotherapeutics [67] and protect cells from drug-induced apoptosis [68-70]. Several studies have found a link between hypoxia and oxidative stress, one of the factors strongly associated with the development of cirrhosis and HCC and therefore an attractive candidate for antitumor therapy [71-73].

Many studies using either overexpression or gene silencing by siRNA have shown possible interactions in cell lines under hypoxia. The relevance of the separate observations and the interplay with other mechanisms need to be validated in a clinical relevant setting.

\section{Chronic Hypoxia: The Additional Dimension}

We hypothesized that, in HCC, there are regions with sustained hypoxia which induces a characteristic gene expression pattern and, further, that the extend of hypoxic gene expression determines the aggressiveness or, more in general, the prognosis.

In our approach, we addressed the problem by starting from an in vitro situation in which the results are not 
clouded by the heterogeneous nature of HCC from patients with different etiologies that would otherwise obscure the underlying processes.

To test our hypothesis, we determined the gene expression in human HCC cells HepG2 under different oxygen conditions $(20,5,2$, and $1 \%)$ and different incubation periods (24 and 72 hours). We tested 6 genes (CCNG2, EGLN3, ERO1L, MAT1A, RCL1, and WDR45L), and a score was determined by RT-PCR based on the relative expression to cells at $20 \% \mathrm{O}_{2}$. For $5 \% \mathrm{O}_{2}$, we did not observe big changes in expression. The most pronounced effect was observed at 72 hours and $2 \% \mathrm{O}_{2}$. Cells cultured in $1 \% \mathrm{O}_{2}$ for 24 hours had a hypoxia score almost equal to $2 \% \mathrm{O}_{2}$ at 72 hours, but this was dominated by only 3 genes (CCNG2, EGLN3, and ERO1L). When HepG2 cells were cultured with $2 \%$ $\mathrm{O}_{2}$ continuously, no extensive cell death was noted, the cell doubling time increased, and the cultures could be passaged by trypsinization for at least 2 months.

The difference in gene expression between acute $(<24$ hours) and prolonged or chronic hypoxia was further demonstrated by immunological staining (Figure 2, semiquantitative quickscore [74]). At $2 \% \mathrm{O}_{2}$, both Hif- $1 \alpha$ and VEGF are upregulated from normoxic conditions $\left(20 \% \mathrm{O}_{2}\right)$ at 72 hours; this was below the peak between 24 and 48 hours that represents the early (acute) reaction to hypoxia.

Using these conditions, we determined the differentially expressed genes in HepG2 cells that were cultured for 72 hours at either $20 \%$ oxygen or in hypoxic conditions at $2 \%$ oxygen by microarray. A total of 37,707 spots showed a representative signal of which $3,592(8 \%)$ with a fold change above 2 and a $P$ value $<.05$ (1,879 upregulated and 1,713 downregulated). A correction for multiple testing was used, and probes with a $P$ value below .01 and a fold change of $>2$ were selected. This resulted in a list of 265 highly significant genes designated as the hypoxic gene set. Next, we used all presently available published datasets [48-50, 75] to investigate the prognostic correlation with our in vitro derived hypoxia gene set. The first three training datasets contained HCCs of 247 patients and the validation dataset 91 HCCs. To test whether the overall expression pattern of these 265 hypoxia genes is significantly related to the prognostic factor considered for each of the three training datasets, the global test of Goeman et al. was used [76]. This resulted in a significant enrichment of the hypoxia gene set for all three training sets $(P$ value .03595 for Boyault, $P$ value $<.00001$ for Lee, and $P$ value .0064 for Wurmbach), keeping only the significant genes with a $z$-score above 1,130 genes remained for the dataset of Lee et al., 43 genes for Boyault et al., and 58 genes for Wurmbach et al. Finally, genes for which the direction of altered expression did not correspond to the direction observed in vivo were removed. With this approach, we were able to downsize our hypoxia gene set to seven genes, the hypoxia signature, found to overlap between the three training datasets (see Figure 3 ).

These 7 genes were used to define the hypoxia score based on the mean (expression ratio UPregulated genes in log base 2)-mean (expression ratio DOWNregulated genes in $\log$ base 2). This hypoxia score was then used to
TABLE 1: List of the 7 hypoxia-related prognostic genes in HCC included in the hypoxia score. CCNG2, EGLN3, ERO1L, and WDR45L will be upregulated under hypoxia in HCC and FGF21, MAT1A, and RCL1 will be downregulated under hypoxia in HCC.

\begin{tabular}{|c|c|c|}
\hline \multicolumn{3}{|c|}{ Genes in the hypoxia signature } \\
\hline Gene & Full name & Function or Pathway \\
\hline CCNG2 & Cyclin G2 & Regulation of the cell cycle \\
\hline EGLN3 & $\begin{array}{l}\text { Egl nine homolog } 1 \text { ( } C \text {. } \\
\text { elegans) }\end{array}$ & $\begin{array}{l}\text { Hif } 1 \alpha \text {-transcription factor } \\
\text { network }\end{array}$ \\
\hline ERO1L & $\begin{array}{l}\text { Endoplasmic reticulum } \\
\text { oxidoreductin-1 L }\end{array}$ & $\begin{array}{l}\text { Protein processing and } \\
\text { oxidoreductase activity }\end{array}$ \\
\hline WDR45L & WDR45-like & $\begin{array}{l}\text { Autophagy and response to } \\
\text { starvation }\end{array}$ \\
\hline FGF21 & Fibroblast growth factor 21 & Hepatic lipid metabolism \\
\hline MAT1A & $\begin{array}{l}\text { Methionine } \\
\text { adenosyltransferase I alpha }\end{array}$ & $\begin{array}{l}\text { Biological oxidation and } \\
\text { metabolism }\end{array}$ \\
\hline RCL1 & $\begin{array}{l}\text { RNA terminal phosphate } \\
\text { cyclase-like } 1\end{array}$ & Ribosome biogenesis \\
\hline
\end{tabular}

classify the patients in the 3 training sets and to calculate the area under the ROC curve (AUC), to assess the predictive performance in all data sets. The hypoxia score based on these seven genes could significantly divide patients with and without vascular invasion (Wurmbach, AUC $88.9 \%$ ), with a FAL-index $>0.128$ and $\leq 0.128$ (Boyault, AUC 72.8\%), and with cluster A and cluster B gene expression (Lee et al., AUC $84.9 \%$ ). The performance of our hypoxia score was confirmed in the Chiang dataset with the BCLC classification as prognostic characteristic. The seven genes significantly separate the BCLC group 0/A/B from C (AUC 91\%) as well as the group $0 / \mathrm{A}$ from $\mathrm{B} / \mathrm{C}$ (AUC $71.5 \%$ ).

The hypoxia score was tested for correlation with survival and recurrence. In a retrospective survival analysis on the 135 patients of the study by Lee et al., if we use a cutoff value of 0.35 for the hypoxia score $(\log$ rank test $P=$ .0018 ), we will be able to demonstrate significant differences in survival in 135 patients with a Kaplan-Meier survival curve (Figure 4(a)). The median survival for patients with a hypoxia score $>0.35(n=42)$ was 307 days, whereas the median survival for patients with a hypoxia score $\leq 0.35$ $(n=93)$ was 1602 days $(P=.002)$. We should make a differentiation between early recurrence $(<2 \mathrm{yrs})$ and late recurrence $(>2 \mathrm{yrs})$ [77, 78]. Recurrence after 2 years is usually a second primary tumor that arises in a cirrhotic liver and has no relation with the first tumor. When early recurrence is the result of dissemination of the primary tumor and tumor characteristics determine the risk of recurrence, our hypoxia score determined on the tumor tissue itself should be able to predict early recurrence. Again, when we use a cutoff of 0.35 for the hypoxia score, the Kaplan-Meier curve shows a significant difference in early recurrence $(P=.005)$ (Figure $4(\mathrm{~b}))$.

In multivariate statistical analysis on 135 patients from the study by Lee et al., using variables such as AFP level $(\leq 300$ versus $>300)$, tumor size $(\leq 5 \mathrm{~cm}$ versus $>5 \mathrm{~cm})$, age $(\leq 65 \mathrm{y}$ versus $>65 \mathrm{y})$, and differentiation grade $(1-2$ versus 3-4), the hypoxia score was a significant variable for both 


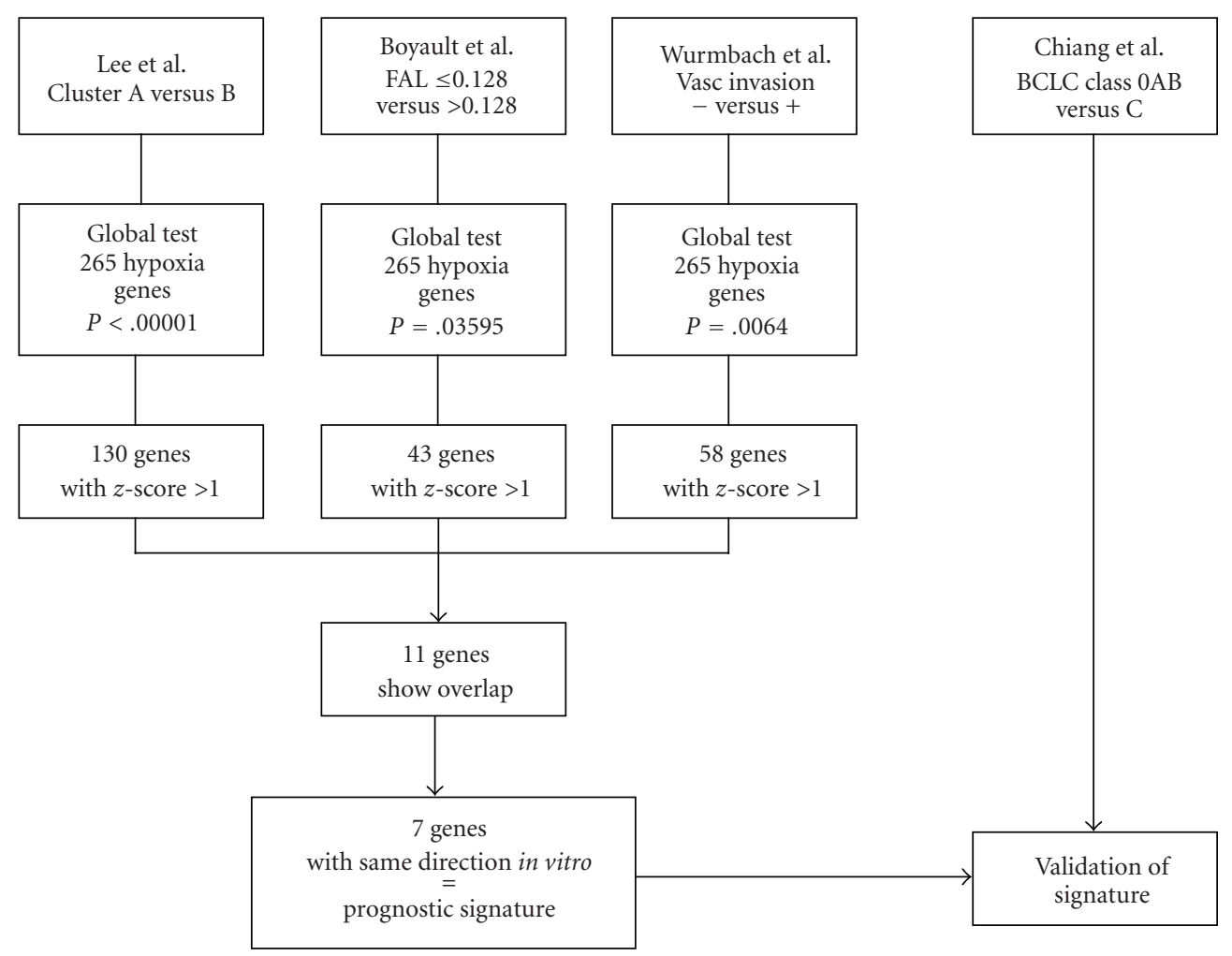

FIGURE 3: Selection procedure of 7-gene prognostic hypoxia gene set. We started with the 265 genes that were identified from the microarray experiments with HepG2 cells. After several selection steps, we could identify a 7-gene set (Table 1) with prognostic value from the studies by Wurmbach et al. [75], Lee et al. [50,51], and Boyault et al. [48]. The prognostic value was subsequently confirmed when we tested this set in the study of Chiang et al. [49].

survival $(P=.02)$ and early recurrence $(P=.008)$ (for full discussion see van Malenstein et al. [79]).

Recently, there was a study by Lui et al. that investigated the activation of b-catenin by chronic hypoxia in HCC [80]. The Wnt/ $\beta$-catenin pathway is identified as an important signaling pathway in cancer and used for the molecular classification of HCC [81]. In around 30\% of HCCs, there is an aberrant expression of $\beta$-catenin $[47,81]$ and $\beta$-catenin has a prominent place in the transcriptome classification by Zucman-Rossi and Boyault [48]. Lui et al. found that exposure for $72 \mathrm{hrs}$ to $1 \% \mathrm{O}_{2}$ induced the upregulation of Hif $1 \alpha$ and $\beta$-catenin protein in PLC/PRF/5 and HepG2 cells but not in other HCC cell lines Hep3B or MHCC97. Under these conditions hypoxia also activated the PI3K/Akt pathway. Both in vitro and in vivo studies using HCC xenograft model showed that $\beta$-catenin depletion through shRNA suppresses the hypoxia-induced increased metastatic potential. This further showed that $\beta$-catenin is critically involved in hypoxia-induced epithelial-mesenchymal transition. There was a shift in expression of epithelial markers (E-cadherin and plakoglobin) to mesenchymal markers (vimentin and $\mathrm{N}$-cadherin).

Using immunohistological staining for $\beta$-catenin and Hifl $\alpha$, they further examined the correlation with prognosis in HCC patients. Hif $1 \alpha$ was found positive in $63 \%$ of patients and was associated with a high incidence of intrahepatic metastasis $(P=.035) . \beta$-catenin was positive in $43.5 \%$ and this correlated with microvascular invasion $(P=.001)$, poor tumor differentiation $(P=.041)$, and high tumor-node metastasis stage $(P=.018)$. Both Hifl $\alpha$ and $\beta$-catenin alone are associated with reduced overall survival and with time to recurrence. Coexpression of Hif $1 \alpha$ and $\beta$-catenin resulted in a higher incidence of metastasis and was associated with a more reduced overall survival and time to recurrence than if only one marker was positive.

Also, Dai et al. has found in a set of 110 HCC patients by analyzing mRNA levels that Hifl $\alpha$ plays an important role in predicting patient's outcome [82]. In the study by Simon et al. [54], the surrounding tissue was investigated and they observed a link between deregulation of Hif $1 \alpha$ and recurrence after curative resection.

Also in models of breast cancer $[83,84]$ and in prostate cells $[85,86]$, the association of chronic hypoxia with EMT transition, resistance to cell death [87], and cancer growth has been demonstrated.

Together with the reduction in blood supply, the supply of nutrients and the removal of waste products will be affected in a tumor, therefore an additional role for the metabolism is expected in cancer possibly in combination with the hypoxia response [88].

\section{Conclusions and Perspectives}

Current treatment options for HCC patients have benefitted from the development of a rational staging system. This has 


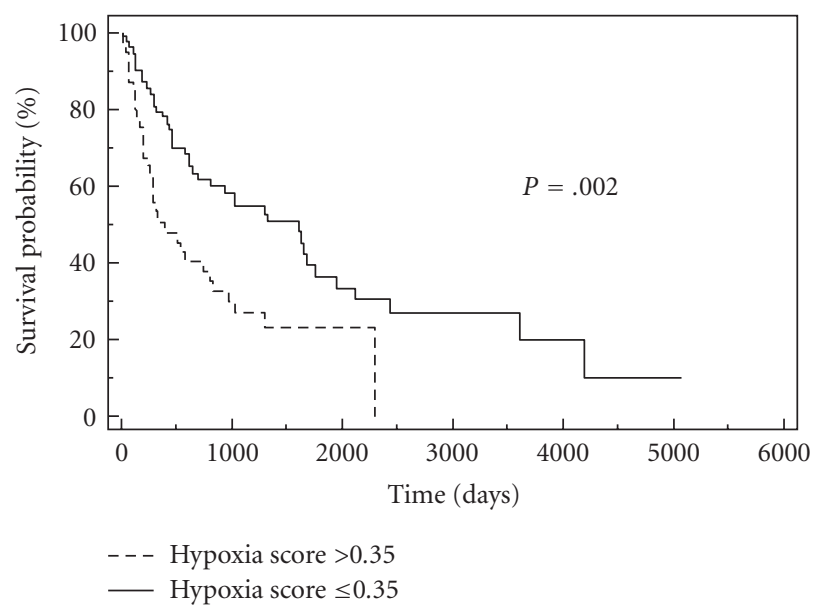

(a)

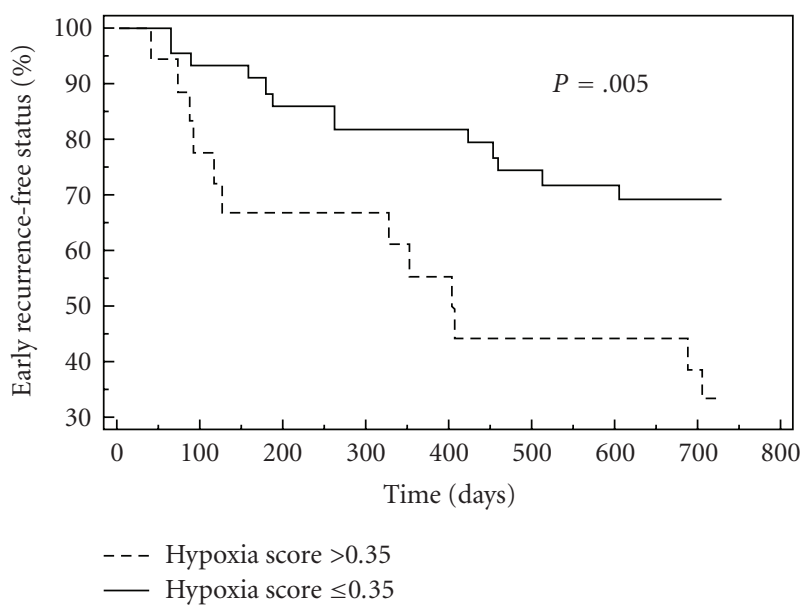

(b)

Figure 4: Survival and early recurrence. (a) Kaplan-Meier survival curve for 135 patients [50, 51]. The hypoxia score was used in a dichotomous matter ( $>0.35$ versus $\leq 0.35$ ). Patients with a score $>0.35$ had a significantly poorer survival. (b) Kaplan-Meier curve for early recurrence $(<2$ yrs $)$ in 135 patients. Again the hypoxia score was used in a dichotomous matter $(>0.35$ versus $\leq 0.35)$. Patients with a score $>0.35$ had a significantly increased risk of early recurrence.

also led the way to an integrated molecular classification based on gene expression and mutational analysis. Due to the nature of this disease, with HCC developing in around $80 \%$ of cases in a cirrhotic lever, systemic therapy in more advanced stages of the disease has had limited success until now.

Gene analysis has already revealed some of the more important molecular pathways and that has helped to identify rational targets for therapy and initiated the development of new classes of drugs.

The relevance of chronic hypoxia has long been overlooked due to the attention for acute events. With the better understanding of neoangiogenesis and the recognition of suboptimal quality of the newly formed blood vessels, chronic hypoxia has now been recognized as a clinical entity. Here, we have three studies that show the importance of gene or protein expression in the chronic situation for patients that may have implications for the prognosis for these patients $[79,80,82]$. These results suggest that chronic hypoxia and the corresponding gene expression are important for the aggressiveness of HCC. The molecular processes need to be studied further which could help to identify on a rational basis additional drug targets. Some overlap has been found between chronic hypoxia and the $\beta$ catenin cluster in the molecular classification; it should be further investigated whether hypoxia should be incorporated in the molecular classification of HCC.

Gene expression under chronic hypoxia has also been linked to drug resistance; perhaps factors responsible for reduced efficacy of anticancer drugs (transport, metabolism) can be identified from these studies.

The integrated approach of strictly controlled cell culture experiments and clinical validation in large sets of patients shows its strength. With the development of a hypoxia gene set not restricted to a single center and that can predict prognosis for individual patients, a promising tool might be at hand. Furthermore, the demonstration of the role of chronic hypoxia in the progression of HCC suggests that it could also play a role in other types of cancer and is worthwhile to be investigated further. In addition, the study by van Malenstein et al. can also be important for other types of cancer for it presents a method to combine the information from different clinical studies in cancer, overcoming methodological differences and validation in the largest available set of clinical and microarray data.

\section{Conflict of Interests}

The authors declared that there is no conflict of interests.

\section{Acknowledgments}

The authors wish to thank Drs. Anneleen Daemen, Olivier Gevaert, and Louis Libbrecht for their contribution to the chronic hypoxia-HCC study. The continued technical support by Mrs. Petra Windmolders and Ingrid Vander Elst at the Liver Research Facility of KU Leuven, Belgium is greatly valued. H.v.Malenstein is a research assistant of the Fund for Scientific Research-Flanders (FWO-Vlaanderen).

\section{References}

[1] D. M. Parkin, F. Bray, J. Ferlay, and P. Pisani, "Global cancer statistics, 2002," Ca: A Cancer Journal for Clinicians, vol. 55, no. 2, pp. 74-108, 2005.

[2] H. B. El-Serag and A. C. Mason, "Rising incidence of hepatocellular carcinoma in the United States," The New England Journal of Medicine, vol. 340, no. 10, pp. 745-750, 1999.

[3] C. Guyot, S. Lepreux, C. Combe et al., "Hepatic fibrosis and cirrhosis: the (myo)fibroblastic cell subpopulations involved," 
The International Journal of Biochemistry and Cell Biology, vol. 38, no. 2, pp. 135-151, 2006.

[4] A. Sangiovanni, E. Del Ninno, P. Fasani et al., "Increased survival of cirrhotic patients with a hepatocellular carcinoma detected during surveillance," Gastroenterology, vol. 126, no. 4, pp. 1005-1014, 2004.

[5] J. M. Llovet, A. Burroughs, and J. Bruix, "Hepatocellular carcinoma," The Lancet, vol. 362, no. 9399, pp. 1907-1917, 2003.

[6] T. Severi, H. Van Malenstein, C. Verslype, and J. F. Van Pelt, "Tumor initiation and progression in hepatocellular carcinoma: risk factors, classification, and therapeutic targets," Acta Pharmacologica Sinica, vol. 31, no. 11, pp. 1409-1420, 2010.

[7] S. M. Hussain, P. E. Zondervan, J. N. M. Ijzermans, S. W. Schalm, R. A. de Man, and G. P. Krestin, "Benign versus malignant hepatic nodules: MR imaging findings with pathologic correlation," Radiographics, vol. 22, no. 5, pp. 1023-1036, 2002.

[8] J. Bruix and M. Sherman, "Management of hepatocellular carcinoma," Hepatology, vol. 42, no. 5, pp. 1208-1236, 2005.

[9] J. M. Llovet, A. M. Di Bisceglie, J. Bruix et al., "Design and endpoints of clinical trials in hepatocellular carcinoma," Journal of the National Cancer Institute, vol. 100, no. 10, pp. 698-711, 2008.

[10] L. Libbrecht, D. Bielen, C. Verslype et al., "Focal lesions in cirrhotic explant livers: pathological evaluation and accuracy of pretransplantation imaging examinations," Liver Transplantation, vol. 8, no. 9, pp. 749-761, 2002.

[11] C. Verslype, E. Van Cutsem, M. Dicato et al., "The management of hepatocellular carcinoma. Current expert opinion and recommendations derived from the 10th World Congress on Gastrointestinal Cancer, Barcelona, 2008," Annals of Oncology, vol. 20, supplement 7, pp. vii1-vii6, 2009.

[12] J. M. Llovet, S. Ricci, V. Mazzaferro et al., "Sorafenib in advanced hepatocellular carcinoma," The New England Journal of Medicine, vol. 359, no. 4, pp. 378-390, 2008.

[13] A. L. Cheng, Y. K. Kang, Z. Chen et al., "Efficacy and safety of sorafenib in patients in the Asia-Pacific region with advanced hepatocellular carcinoma: a phase III randomised, doubleblind, placebo-controlled trial," The Lancet Oncology, vol. 10, no. 1, pp. 25-34, 2009.

[14] I. P. Witz, "The tumor microenvironment: the making of a paradigm," Cancer Microenvironment, vol. 2, no. 1, pp. S9-S17, 2009.

[15] A. Mantovani, P. Allavena, A. Sica, and F. Balkwill, "Cancerrelated inflammation," Nature, vol. 454, no. 7203, pp. 436-444, 2008.

[16] R. Kalluri and M. Zeisberg, "Fibroblasts in cancer," Nature Reviews Cancer, vol. 6, no. 5, pp. 392-401, 2006.

[17] A. Mazzocca, E. Fransvea, F. Dituri, L. Lupo, S. Antonaci, and G. Giannelli, "Down-regulation of connective tissue growth factor by inhibition of transforming growth factor $\beta$ blocks the tumor-stroma cross-talk and tumor progression in hepatocellular carcinoma," Hepatology, vol. 51, no. 2, pp. 523534,2010

[18] F. Van Zijl, M. Mair, A. Csiszar et al., "Hepatic tumor-stroma crosstalk guides epithelial to mesenchymal transition at the tumor edge," Oncogene, vol. 28, no. 45, pp. 4022-4033, 2009.

[19] K. Sasaki, S. Natsugoe, S. Ishigami et al., "Significance of Twist expression and its association with E-cadherin in esophageal squamous cell carcinoma," Journal of Experimental \& Clinical Cancer Research, vol. 28, no. 1, p. 158, 2009.

[20] M. Iwatsuki, K. Mimori, T. Fukagawa et al., "The clinical significance of vimentin-expressing gastric cancer cells in bone marrow," Annals of Surgical Oncology, vol. 17, no. 9, pp. 25262533, 2010.

[21] T. Jäger, M. Becker, A. Eisenhardt et al., "The prognostic value of cadherin switch in bladder cancer," Oncology Reports, vol. 23, no. 4, pp. 1125-1132, 2010.

[22] A. Soltermann, V. Tischler, S. Arbogast et al., "Prognostic significance of epithelial-mesenchymal and mesenchymalepithelial transition protein expression in non-small cell lung cancer," Clinical Cancer Research, vol. 14, no. 22, pp. 74307437, 2008.

[23] J. M. M. Cates, R. H. Byrd, L. E. Fohn, A. D. Tatsas, M. K. Washington, and C. C. Black, "Epithelial-mesenchymal transition markers in pancreatic ductal adenocarcinoma," Pancreas, vol. 38, no. 1, pp. e1-e6, 2009.

[24] M. H. Yang, C. L. Chen, G. Y. Chau et al., "Comprehensive analysis of the independent effect of twist and snail in promoting metastasis of hepatocellular carcinoma," Hepatology, vol. 50, no. 5, pp. 1464-1474, 2009.

[25] R. F. Niu, L. Zhang, G. M. Xi et al., "Up-regulation of twist induces angiogenesis and correlates with metastasis in hepatocellular carcinoma," Journal of Experimental \& Clinical Cancer Research, vol. 26, no. 3, pp. 385-394, 2007.

[26] L. Chen, T. H. M. Chan, Y. F. Yuan et al., "CHD1L promotes hepatocellular carcinoma progression and metastasis in mice and is associated with these processes in human patients," Journal of Clinical Investigation, vol. 120, no. 4, pp. 1178-1191, 2010.

[27] F. van Zijl, G. Zulehner, M. Petz et al., "Epithelialmesenchymal transition in hepatocellular carcinoma," Future Oncology, vol. 5, no. 8, pp. 1169-1179, 2009.

[28] T. K. Lee, R. T. P. Poon, A. P. Yuen et al., "Twist overexpression correlates with hepatocellular carcinoma metastasis through induction of epithelial-mesenchymal transition," Clinical Cancer Research, vol. 12, no. 18, pp. 5369-5376, 2006.

[29] C. Cicchini, I. Laudadio, F. Citarella et al., "TGF $\beta$-induced EMT requires focal adhesion kinase (FAK) signaling," Experimental Cell Research, vol. 314, no. 1, pp. 143-152, 2008.

[30] N. Matsuo, H. Shiraha, T. Fujikawa et al., "Twist expression promotes migration and invasion in hepatocellular carcinoma," BMC Cancer, vol. 9, article 240, 2009.

[31] M. I. Kokkinos, R. Wafai, M. K. Wong, D. F. Newgreen, E. W. Thompson, and M. Waltham, "Vimentin and epithelial-mesenchymal transition in human breast cancerobservations in vitro and in vivo," Cells Tissues Organs, vol. 185, no. 1-3, pp. 191-203, 2007.

[32] M. Zeisberg and E. G. Neilson, "Biomarkers for epithelialmesenchymal transitions," Journal of Clinical Investigation, vol. 119, no. 6, pp. 1429-1437, 2009.

[33] C. D. Venkov, A. J. Link, J. L. Jennings et al., "A proximal activator of transcription in epithelial-mesenchymal transition," Journal of Clinical Investigation, vol. 117, no. 2, pp. 482-491, 2007.

[34] J. Gotzmann, A. N. M. Fischer, M. Zojer et al., "A crucial function of PDGF in TGF- $\beta$-mediated cancer progression of hepatocytes," Oncogene, vol. 25, no. 22, pp. 3170-3185, 2006.

[35] C. Y. Lin, C. J. Lin, K. H. Chen, J. C. Wu, S. H. Huang, and S. M. Wang, "Macrophage activation increases the invasive properties of hepatoma cells by destabilization of the adherens junction," FEBS Letters, vol. 580, no. 13, pp. 3042-3050, 2006.

[36] W. Ding, H. You, H. Dang et al., "Epithelial-to-mesenchymal transition of murine liver tumor cells promotes invasion," Hepatology, vol. 52, no. 3, pp. 945-953, 2010.

[37] S. Z. Yang, L. D. Zhang, YI. Zhang et al., "HBx protein induces EMT through c-Src activation in SMMC-7721 hepatoma cell 
line," Biochemical \& Biophysical Research Communications, vol. 382, no. 3, pp. 555-560, 2009.

[38] S. Battaglia, N. Benzoubir, S. Nobilet et al., "Liver cancerderived hepatitis $\mathrm{C}$ virus core proteins shift TGF-beta responses from tumor suppression to epithelial-mesenchymal transition," PloS One, vol. 4, no. 2, Article ID e4355, 2009.

[39] J. Kim, S. J. Hong, J. Y. Park et al., "Epithelial—mesenchymal transition gene signature to predict clinical outcome of hepatocellular carcinoma," Cancer Science, vol. 101, no. 6, pp. 1521-1528, 2010.

[40] F. Dayan, N. M. Mazure, M. C. Brahimi-Horn, and J. Pouysségur, "A dialogue between the hypoxia-inducible factor and the tumor microenvironment," Cancer Microenvironment, vol. 1, no. 1, pp. 53-68, 2008.

[41] K. Murata, H. Suzuki, H. Okano, T. Oyamada, Y. Yasuda, and A. Sakamoto, "Hypoxia-induced des- $\gamma$-carboxy prothrombin production in hepatocellular carcinoma," International Journal of Oncology, vol. 36, no. 1, pp. 161-170, 2010.

[42] B. L. Copple, "Hypoxia stimulates hepatocyte epithelial to mesenchymal transition by hypoxia-inducible factor and transforming growth factor- $\beta$-dependent mechanisms," Liver International, vol. 30, no. 5, pp. 669-682, 2010.

[43] W. Yan, Y. Fu, D. Tian et al., "PI3 kinase/Akt signaling mediates epithelial-mesenchymal transition in hypoxic hepatocellular carcinoma cells," Biochemical \& Biophysical Research Communications, vol. 382, no. 3, pp. 631-636, 2009.

[44] V. H. Haase, "Oxygen regulates epithelial-to-mesenchymal transition: insights into molecular mechanisms and relevance to disease," Kidney International, vol. 76, no. 5, pp. 492-499, 2009.

[45] S. Sun, X. Ning, Y. Zhang et al., "Hypoxia-inducible factor- $1 \alpha$ induces Twist expression in tubular epithelial cells subjected to hypoxia, leading to epithelial-to-mesenchymal transition," Kidney International, vol. 75, no. 12, pp. 1278-1287, 2009.

[46] S. Cannito, E. Novo, A. Compagnone et al., "Redox mechanisms switch on hypoxia-dependent epithelial-mesenchymal transition in cancer cells," Carcinogenesis, vol. 29, no. 12, pp. 2267-2278, 2008.

[47] A. Villanueva, P. Newell, D. Y. Chiang, S. L. Friedman, and J. M. Llovet, "Genomics and signaling pathways in hepatocellular carcinoma," Seminars in Liver Disease, vol. 27, no. 1, pp. 55-76, 2007.

[48] S. Boyault, D. S. Rickman, A. de Reyniès et al., "Transcriptome classification of HCC is related to gene alterations and to new therapeutic targets," Hepatology, vol. 45, no. 1, pp. 42-52, 2007.

[49] D. Y. Chiang, A. Villanueva, Y. Hoshida et al., "Focal gains of VEGFA and molecular classification of hepatocellular carcinoma," Cancer Research, vol. 68, no. 16, pp. 6779-6788, 2008.

[50] J. S. Lee, J. Heo, L. Libbrecht et al., "A novel prognostic subtype of human hepatocellular carcinoma derived from hepatic progenitor cells," Nature Medicine, vol. 12, no. 4, pp. 410-416, 2006.

[51] J. S. Lee, IN. S. Chu, J. Heo et al., "Classification and prediction of survival in hepatocellular carcinoma by gene expression profiling," Hepatology, vol. 40, no. 3, pp. 667-676, 2004.

[52] Y. Hoshida, A. Villanueva, M. Kobayashi et al., "Gene expression in fixed tissues and outcome in hepatocellular carcinoma," The New England Journal of Medicine, vol. 359, no. 19, pp. 1995-2004, 2008.

[53] A. Budhu, M. Forgues, Q. H. Ye et al., "Prediction of venous metastases, recurrence, and prognosis in hepatocellular carcinoma based on a unique immune response signature of the liver microenvironment," Cancer Cell, vol. 10, no. 2, pp. 99$111,2006$.

[54] F. Simon, M. Bockhorn, C. Praha et al., "Deregulation of HIF1-alpha and hypoxia-regulated pathways in hepatocellular carcinoma and corresponding non-malignant liver tissueinfluence of a modulated host stroma on the prognosis of HCC," Langenbeck's Archives of Surgery, vol. 395, no. 4, pp. 395-405, 2010.

[55] J. F. Dufour and P. Johnson, "Liver cancer: from molecular pathogenesis to new therapies. Summary of the EASL single topic conference," Journal of Hepatology, vol. 52, no. 2, pp. 296-304, 2010.

[56] E. H. Gort, A. J. Groot, E. van der Wall, P. J. van Diest, and M. A. Vooijs, "Hypoxic regulation of metastasis via hypoxiainducible factors," Current Molecular Medicine, vol. 8, no. 1, pp. 60-67, 2008.

[57] G. L. Semenza, "Targeting HIF-1 for cancer therapy," Nature Reviews Cancer, vol. 3, no. 10, pp. 721-732, 2003.

[58] J. M. Brown and W. R. Wilson, "Exploiting tumour hypoxia in cancer treatment," Nature Reviews Cancer, vol. 4, no. 6, pp. 437-447, 2004.

[59] J. M. Brown and A. J. Giaccia, "The unique physiology of solid tumors: opportunities (and problems) for cancer therapy," Cancer Research, vol. 58, no. 7, pp. 1408-1416, 1998.

[60] K. R. Kim, H. E. Moon, and K. W. Kim, "Hypoxia-induced angiogenesis in human hepatocellular carcinoma," Journal of Molecular Medicine, vol. 80, no. 11, pp. 703-714, 2002.

[61] J. Folkman, P. Hahnfeldt, and L. Hlatky, "Cancer: looking outside the genome," Nature Reviews Molecular Cell Biology, vol. 1, no. 1, pp. 76-79, 2000.

[62] M. C. Brahimi-Horn, J. Chiche, and J. Pouysségur, "Hypoxia and cancer," Journal of Molecular Medicine, vol. 85, no. 12, pp. 1301-1307, 2007.

[63] T. Fink, P. Ebbesen, and V. Zachar, "Quantitative gene expression profiles of human liver-derived cell lines exposed to moderate hypoxia," Cellular Physiology and Biochemistry, vol. 11, no. 2, pp. 105-114, 2001.

[64] A. Vengellur, J. M. Phillips, J. B. Hogenesch, and J. J. LaPres, "Gene expression profiling of hypoxia signaling in human hepatocellular carcinoma cells," Physiological Genomics, vol. 22, pp. 308-318, 2005.

[65] L. A. Sonna, M. L. Cullivan, H. K. Sheldon, R. E. Pratt, and C. M. Lilly, "Effect of hypoxia on gene expression by human hepatocytes (HepG2)," Physiological Genomics, vol. 12, pp. 195-207, 2003.

[66] X. Zhao, J. Li, J. Zhuo, and L. Cai, "Reexpression of ARHI inhibits tumor growth and angiogenesis and impairs the mTOR/VEGF pathway in hepatocellular carcinoma," Biochemical \& Biophysical Research Communications, vol. 403, no. 3-4, pp. 417-421, 2010.

[67] H. Zhu, X. P. Chen, S. F. Luo, J. Guan, W. G. Zhang, and B. X. Zhang, "Involvement of hypoxia-inducible factor-1- $\alpha$ in multidrug resistance induced by hypoxia in HepG2 cells," Journal of Experimental \& Clinical Cancer Research, vol. 24, no. 4, pp. 565-574, 2005.

[68] A. Sermeus, J. P. Cosse, M. Crespin et al., "Hypoxia induces protection against etoposide-induced apoptosis: molecular profiling of changes in gene expression and transcription factor activity," Molecular Cancer, vol. 7, article 27, 2008.

[69] J.-P. Cosse, G. Rommelaere, N. Ninane, T. Arnould, and C. Michiels, "BNIP3 protects HepG2 cells against etoposideinduced cell death under hypoxia by an autophagyindependent pathway," Biochemical Pharmacology, vol. 80, no. 8, pp. 1160-1169, 2010. 
[70] E. U. Jung, J. H. Yoon, Y. J. Lee et al., "Hypoxia and retinoic acid-inducible NDRG1 expression is responsible for doxorubicin and retinoic acid resistance in hepatocellular carcinoma cells," Cancer Letters, vol. 298, no. 1, pp. 9-15, 2010.

[71] E. Tak, S. Lee, J. Lee et al., "Human carbonyl reductase 1 upregulated by hypoxia renders resistance to apoptosis in hepatocellular carcinoma cells," Journal of Hepatology, vol. 54, no. 2, pp. 328-339, 2011.

[72] S. W. Jusman, A. Halim, S. I. Wanandi, and M. Sadikin, "Expression of hypoxia-inducible factor-1alpha (HIF-1alpha) related to oxidative stress in liver of rat-induced by systemic chronic normobaric hypoxia," Acta medica Indonesiana, vol. 42, no. 1, pp. 17-23, 2010.

[73] J. E. Klaunig, L. M. Kamendulis, and B. A. Hocevar, "Oxidative stress and oxidative damage in carcinogenesis," Toxicologic Pathology, vol. 38, no. 1, pp. 96-109, 2010.

[74] S. Detre, G. S. Jotti, and M. Dowsett, "A 'quickscore' method for immunohistochemical semiquantitation: validation for oestrogen receptor in breast carcinomas," Journal of Clinical Pathology, vol. 48, no. 9, pp. 876-878, 1995.

[75] E. Wurmbach, Y. B. Chen, G. Khitrov et al., "Genome-wide molecular profiles of $\mathrm{HCV}$-induced dysplasia and hepatocellular carcinoma," Hepatology, vol. 45, no. 4, pp. 938-947, 2007.

[76] J. J. Goeman, S. van de Geer, F. de Kort, and H. C. van Houwellingen, "A global test for groups fo genes: testing association with a clinical outcome," Bioinformatics, vol. 20, no. 1, pp. 93-99, 2004.

[77] A. Cucchetti, F. Piscaglia, E. Caturelli et al., "Comparison of recurrence of hepatocellular carcinoma after resection in patients with cirrhosis to its occurrence in a surveilled cirrhotic population," Annals of Surgical Oncology, vol. 16, no. 2, pp. 413-422, 2009.

[78] H. Imamura, Y. Matsuyama, E. Tanaka et al., "Risk factors contributing to early and late phase intrahepatic recurrence of hepatocellular carcinoma after hepatectomy," Journal of Hepatology, vol. 38, no. 2, pp. 200-207, 2003.

[79] H. van Malenstein, O. Gevaert, L. Libbrecht et al., "A sevengene set associated with chronic hypoxia of prognostic importance in hepatocellular carcinoma," Clinical Cancer Research, vol. 16, no. 16, pp. 4278-4288, 2010.

[80] L. Liu, X. D. Zhu, W. Q. Wang et al., "Activation of $\beta$ catenin by hypoxia in hepatocellular carcinoma contributes to enhanced metastatic potential and poor prognosis," Clinical Cancer Research, vol. 16, no. 10, pp. 2740-2750, 2010.

[81] J. M. Llovet and J. Bruix, "Molecular targeted therapies in hepatocellular carcinoma," Hepatology, vol. 48, no. 4, pp. 1312-1327, 2008.

[82] C.-X. Dai, Q. Gao, S.-J. Qiu et al., "Hypoxia-inducible factor1 alpha, in association with inflammation, angiogenesis and MYC, is a critical prognostic factor in patients with HCC after surgery," BMC Cancer, vol. 9, article 418, 2009.

[83] T. M. Kalliomäki, G. McCallum, P. G. Wells, and R. P. Hill, "Progression and metastasis in a transgenic mouse breast cancer model: effects of exposure to in vivo hypoxia," Cancer Letters, vol. 282, no. 1, pp. 98-108, 2009.

[84] E. Louie, S. Nik, J.-S. Chen et al., "Identification of a stemlike cell population by exposing metastatic breast cancer cell lines to repetitive cycles of hypoxia and reoxygenation," Breast Cancer Research, vol. 12, no. 6, article R94, 2010.

[85] L. Terraneo, P. Bianciardi, A. Caretti, R. Ronchi, and M. Samaja, "Chronic systemic hypoxia promotes LNCaP prostate cancer growth in vivo," Prostate, vol. 70, no. 11, pp. 1243-1254, 2010.

[86] J. A. Watson, C. J. Watson, A. M. McCrohan et al., "Generation of an epigenetic signature by chronic hypoxia in prostate cells,"
Human Molecular Genetics, vol. 18, no. 19, pp. 3594-3604, 2009.

[87] A. Ginouvès, K. Ilc, N. Macías, J. Pouysségur, and E. Berra, "PHDs overactivation during chronic hypoxia "desensitizes" $\mathrm{HIF} \alpha$ and protects cells from necrosis," Proceedings of the National Academy of Sciences of the United States of America, vol. 105, no. 12, pp. 4745-4750, 2008.

[88] R. G. Bristow and R. P. Hill, "Hypoxia and metabolism: hypoxia, DNA repair and genetic instability," Nature Reviews Cancer, vol. 8, no. 3, pp. 180-192, 2008. 


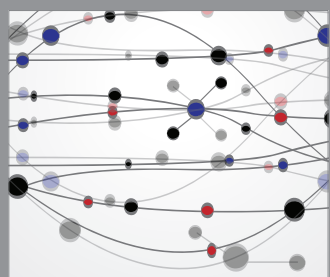

The Scientific World Journal
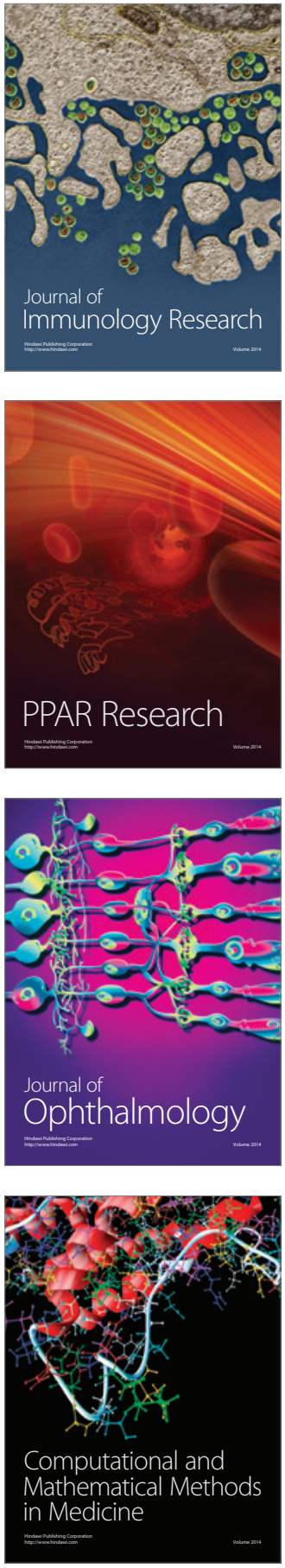

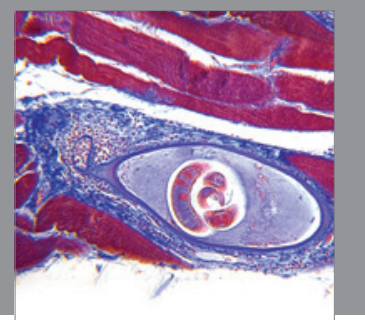

Gastroenterology

Research and Practice
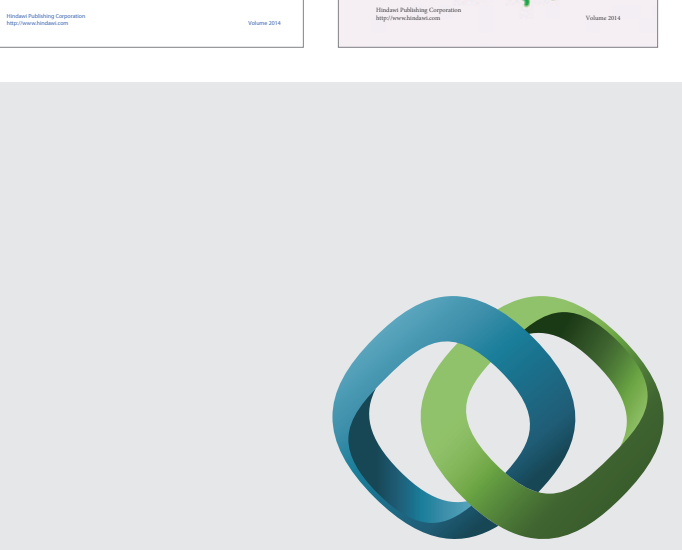

\section{Hindawi}

Submit your manuscripts at

http://www.hindawi.com
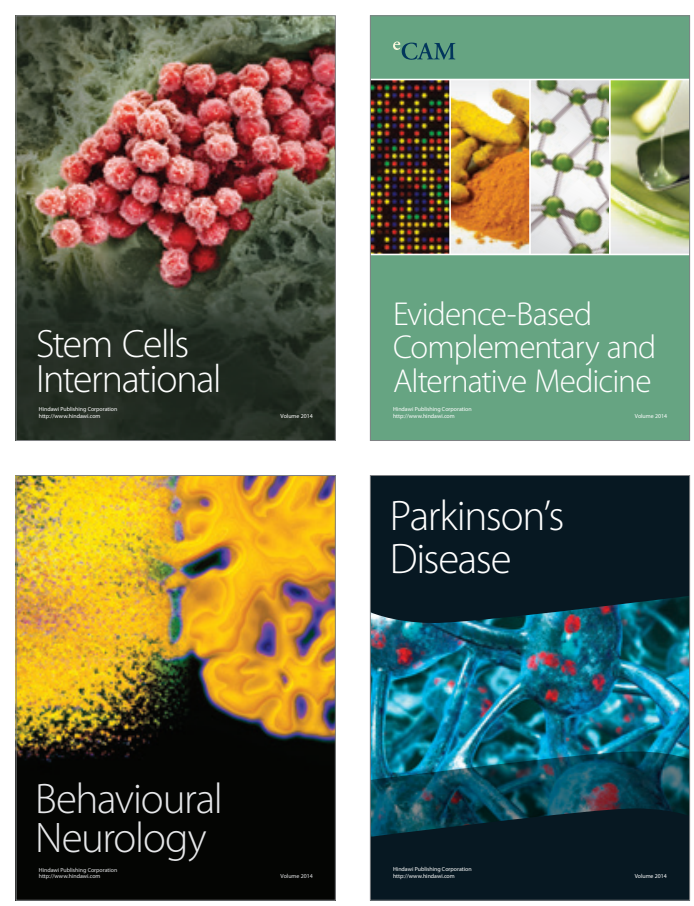

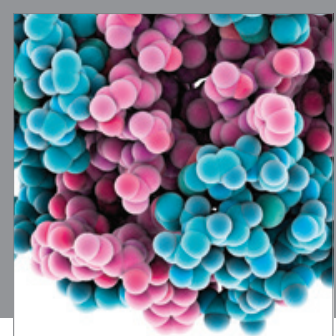

Journal of
Diabetes Research

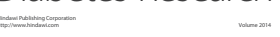

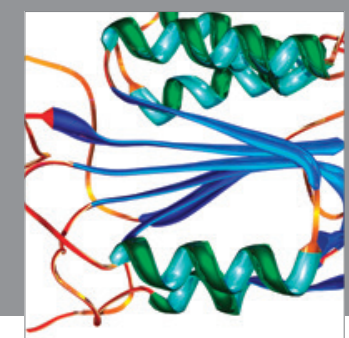

Disease Markers
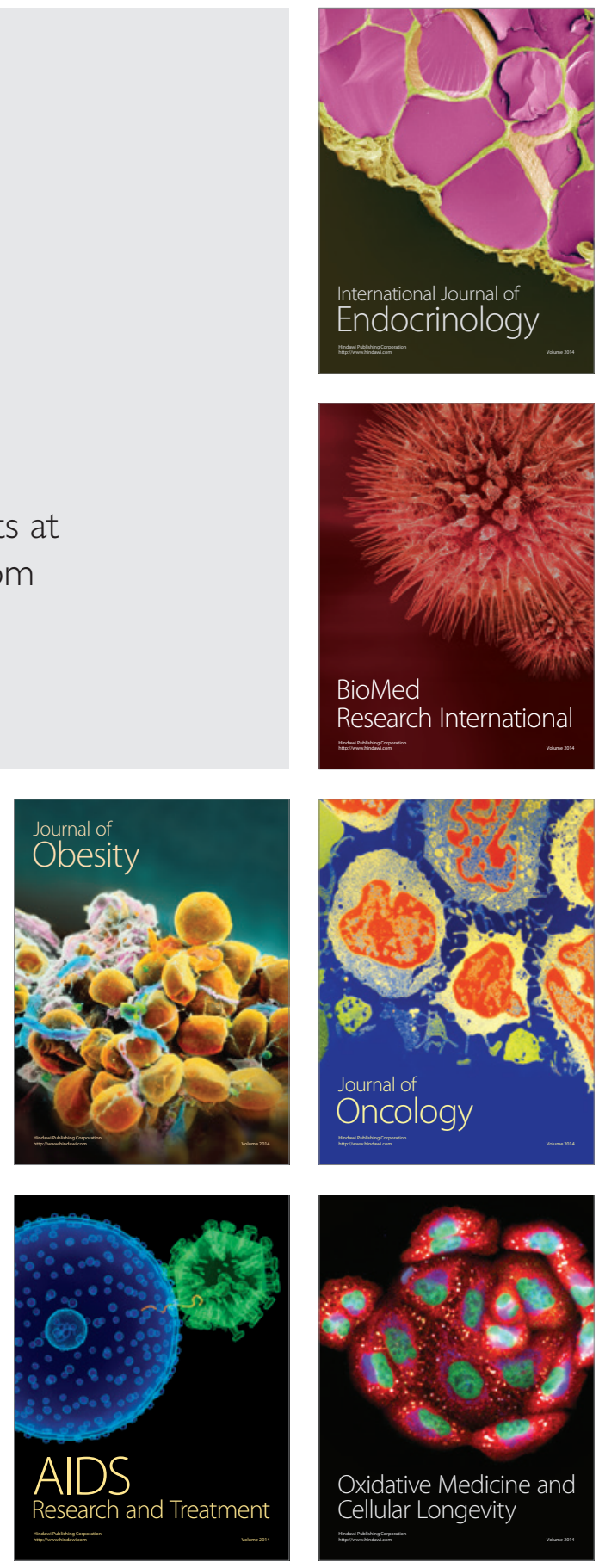